\title{
Four Cases of Allergic and/or Autoimmune Diseases That Completely Recovered After Repetitions of Intradermal Injections with a Non-Specific Antigen Preparation
}

\section{Kimihiko Okazaki*}

Okazaki Medical Clinic, Ukyoku Kyoto, Japan

*Corresponding author: Kimihiko Okazaki, Okazaki Medical Clinic, Ukyoku Kyoto, Japan, Tel: +81-75-753-7531; E-mail: ma13081x@ma1.seikyou.ne.jp

Received date: August 22, 2017; Accepted date: August 29, 2017; Published date: September 06, 2017

Copyright: ( 2017 Okazaki K. This is an open-access article distributed under the terms of the Creative Commons Attribution License, which permits unrestricted use, distribution, and reproduction in any medium, provided the original author and source are credited.

Citation: Okazaki K (2017) Four Cases of Allergic and/or Autoimmune Diseases That Completely Recovered After Repetitions of Intradermal Injections with a NonSpecific Antigen Preparation. J Allergy Ther 8: 264. doi:10.4172/2155-6121.1000264

Keywords: Autoimmune Diseases; Allergy; Immunology; Cytolytic

\section{Case Report}

According to the traditional concept of the contemporary Immunology, neither autoimmune diseases nor allergic diseases can be cured completely. Nevertheless, a fortunate coincidence led me to discovery of a novel concept that eliminations of the causes of these diseases are possible. In other words, combinations of pathogenic antibodies with responsible cells, namely, cytolytic $\mathrm{T}$ lymphocytes in cases of autoimmune diseases and mast cells in cases of allergic diseases, can be decomposed by replacing the pathogenic antibodies with non-specific antibodies. In more detail, intradermal injections with a non-specific antigen preparation induce production of nonspecific antibodies in the body of the patient. Repetitions of the injections bring about an accumulation of them. Accumulated nonspecific antibodies will occupy most of the receptors on the surface of responsible cells. When the accumulation reaches the sufficient level, virtually no pathogenic antibodies would remain on the receptors. That is, no causes of the diseases remain. Naturally, where there is no cause, there is no disease. Details are demonstrated elsewhere [1].

\section{Case 1}

A 43-year-old woman (E.M.) visited my clinic on July 24, 2013. She told me that she had had cedar and cypress pollen allergy since a little longer than 20 years ago and that she had had morning stiffness in her left second and third fingers since 3 months ago. I diagnosed her as pollen allergy and an early stage of rheumatoid arthritis. I gave her intradermal injections with $0.1 \mathrm{ml}$ of $10,000,000$-fold with saline diluted Neurotropin; a product of Nippon Zohki Pharmaceutical Company(Osaka), consisting of an extract of rabbit skin inflamed by inoculation of Vaccinia virus, at 2 10 day-intervals during the period from July 24, 2013 to January 26, 2014. The total number of the injections was 28. As of August 21, 2017, she still has a mild coryza in early mornings breathing fresh and cool air, otherwise, she is enjoying a healthy life.

\section{Case 2}

An 11-year-old girl (A.M.) visited my clinic on July 24, 2013. She told me that she has had a cedar-pollen allergy for 18 months. I gave her intradermal injections with $0.1 \mathrm{ml}$ of 10,000 -fold diluted Neurotropin at 2 10 day intervals for 18 months. The total number of injections was 40. As of August 21,2017, she is quite healthy except for intermittent mild coryza.

\section{Case 3}

A 15 -year-old boy (R.M.) visited my clinic on July 31, 2013. He told me that he has had cedar pollen and a weed pollen allergy for 5 years. I gave him intradermal injections with $0.1 \mathrm{ml}$ of 100,000- fold diluted Neurotropin at 3 14 day intervals for 18 months. The total number of injections was 39. As of August 21, 2017, he has no symptom of allergy.

\section{Case 4}

A 56-year-old woman (A.Y.) visited my clinic on December 8, 2014. She told me that she had a systemic eruption after applying hair-dye about a month earlier, which lasted until her visiting my clinic. I diagnosed her as hair-dye allergy and gave her an intradermal injection with $0.1 \mathrm{ml}$ of 10 to the 30 -fold diluted Neurotropin at 2months intervals 4 times. As of August 24, 2017, she is free from any allergic symptoms.

\section{Reference}

1. Okazaki K (2009) Therapeutic Significance of Non-Specific Antigens as Anti-Allergic and Anti- Autoimmune Agents. Pharmacometrics 76: 105-107. 